\title{
Lava channel formation during the 2001 eruption on Mount Etna: evidence for mechanical erosion
}

\author{
Carmelo Ferlito ${ }^{1}$ and Jens Siewert ${ }^{2}$ \\ ${ }^{1}$ Dipartimento Scienze Geologiche, Università di Catania, I-95125 Catania, Italy \\ ${ }^{2}$ MATIS-INFM \& Dipartimento di Metodologie Fisiche e Chimiche (DMFCI), \\ viale A. Doria 6, 95125 Catania, Italy
}

\begin{abstract}
We report the direct observation of a peculiar lava channel that was formed near the base of a parasitic cone during the 2001 eruption on Mount Etna. Erosive processes by flowing lava are commonly attributed to thermal erosion. However, field evidence strongly suggests that models of thermal erosion cannot explain the formation of this channel. Here, we put forward the idea that the essential erosion mechanism was abrasive wear. By applying a simple model from tribology we demonstrate that the available data agree favorably with our hypothesis. Consequently, we propose that erosional processes resembling the wear phenomena in glacial erosion are possible in a volcanic environment.

PACS numbers: 46.55.+d, 81.40.Pq, 91.40.Hw, 92.40.Gc
\end{abstract}


Introduction - Formation of lava channels is usually discussed in terms of thermal or thermomechanical erosion [1, 2, 3, 4, 5, 6, 17]. The basic idea is that heat is transferred from the hot flowing lava to the cold substrate, thereby melting it and washing it out. Thermal erosion by lava has been studied quantitatively both for turbulent [1, 3, 4, 5] and for laminar flows [2, 6, 7]. The physics underlying the quantitative description is that of heat transport in liquids and solids combined with fluid dynamics. The idea of thermal erosion in the formation of lava channels is supported by field evidence only in few examples [8, 9, 10, 11, 12], e.g., in archaean komatiite lavas 8], in African carbonatites [9, 10], or in basaltic lava tubes of Kilauea volcano (Hawaii) [11].

In the picture of thermal erosion, the relevance of mechanical processes appears to be secondary being mainly limited to the removal of the melted material. However, it has been noted that mechanical effects may enhance the erosion rates [6, 12]. Very recently, Williams et al. have reported geochemical evidence for mechanical erosion in the Cave Basalt of Mount St. Helens where a basaltic flow eroded an unconsolidated pyroclastic substrate [13]. To our knowledge, this is the only example in the literature in which a dominant role of mechanical erosion of lava has been inferred (aspects of models for mechanical erosion have been considered theoretically before [14]). Therefore, the direct observation of the formation of a lava channel whose origin cannot be explained by thermal erosion is of considerable interest. Such an observation may help to develop new and more complete models for the explanation of the field data elsewhere. Moreover, it generates interesting cross-links to other branches of geology where a variety of erosion models are discussed.

The starting point of this work was the formation of an uncommon channel during the 2001 eruption on Mount Etna which provides field evidence for purely mechanical erosion of lava flow. First, we briefly describe the morphological details of the channel and the history of the eruption (see also Ref. 15]). An estimate of the heat transfer required for the formation of the channel basically rules out thermal erosion as the essential process. Then, we will discuss the possibility of erosion due to abrasive wear and demonstrate that this hypothesis may account also quantitatively for the field observations. We will conclude the discussion of our model by deriving some theoretical predictions and compare them to those for thermal erosion.

Description of the Laghetto channel-The channel, informally named Laghetto, is located on the upper southern flank of Mount Etna volcano at an elevation of $2560 \mathrm{~m}$ a.s.l. (see Fig. 
1). It is roughly linear, of rectangular-shaped cross section and is incised on a lava slope gently inclined $\left(5^{\circ}-10^{\circ}\right)$. Its axis is oriented E-W. It is $220 \mathrm{~m}$ long and has a width between 8 and $16 \mathrm{~m}$. Maximum depth is $6 \mathrm{~m}$, the floor of the channel is covered by lava. The banks consist of lava flows emitted between July 25th and 30th. They are formed of up to 11 thin lava layers, each about 0.2 to $0.3 \mathrm{~m}$ thick (cf. Fig. 2). The eroded volume is about $220 \mathrm{~m} \times$ $12 \mathrm{~m} \times 4 \mathrm{~m} \approx 10,000 \mathrm{~m}^{3}$. Note that this volume is underestimated since part of the channel is filled with the eroding lava.

Description of the eruptive event - The eruption started on July 19th forming a crater in the area of Piano del Lago. In the first week, the eruption (closely observed by the authors) gave rise only to phreatomagmatic explosions and lava fountains. From July 25th for the 5 following days lava was erupted from the vent. The relatively low lava output (probably $<5 \mathrm{~m}^{3} / \mathrm{s}$ ) was not constant but intermittent thus originating a number of thin and short (average length $<500 \mathrm{~m}$ ) lava flows.

In the evening of July 30th we witnessed an abrupt change in the lava output. A huge lava flow (10 to $20 \mathrm{~m}$ high) was emitted. The lava flowed (in laminar motion) for about 12 hours reaching the area of Rifugio Sapienza, $2000 \mathrm{~m}$ down valley. Near the vent the lava flowed on a bedrock formed by the still hot and soft thin lava layers of the preceding days. In these hours the bedrock was eroded and the following morning the Laghetto channel was there. After this last flow, lava emission at the Laghetto vent ceased. Therefore, the channel was not buried under subsequent lavas. It is this fortunate circumstance that allowed us to study the phenomenon and measure the channel before it was partially covered by sand and ash fall of the flank eruption of October 2002.

Thermal erosion hypothesis - Let us assume that the channel was thermally eroded. This implies that the lava flow had to supply (at least) the energy $\Delta \mathcal{E}$ to melt the material originally contained in the channel, i.e., the volume $W_{v} \approx 10^{4} \mathrm{~m}^{3}$

$$
\Delta \mathcal{E}=\rho W_{v} \cdot\left(L+c\left(T_{m}-T_{\text {subst }}\right)\right) .
$$

Here $c=1.4 \cdot 10^{3} \mathrm{~J} /(\mathrm{kg} \mathrm{K})$ is the specific heat of the lava substrate (calculated for the specific lava composition using MELTS [16]), $T_{m} \approx 1050^{\circ} \mathrm{C}$ is a lower limit for the onset of melting, and $T_{\text {subst }} \sim 900^{\circ} \mathrm{C}$ denotes the likely substrate temperature. With a lava density of $\rho=2800 \mathrm{~kg} / \mathrm{m}^{3}$ and a latent heat of $L=0.5 \cdot 5.0 \cdot 10^{5} \mathrm{~J} / \mathrm{kg}$ for an assumed $50 \%$ cristallinity (using typical values for basaltic lavas [6, 7, 13]), about $1.3 \times 10^{13}$ Joule were required. 
The eroding lava flow had a total length $s \approx 2000 \mathrm{~m}$ (the distance between vent and the Rifugio Sapienza area) and an average height $h_{\mathrm{fl}} \sim 10 \mathrm{~m}$. Typically, Mount Etna lavas are rich in phenocrysts (i.e., they are erupted partially solidified) and their temperature $\left(T_{l} \sim 1050-1100^{\circ} \mathrm{C}\right)$ is close to the solidus point.

Assuming an excessive temperature difference $T_{l}-T_{m} \sim 50 \mathrm{~K}$, the lava flow (approx. $240,000 \mathrm{~m}^{3}$ ) could provide at most $4.7 \times 10^{13}$ Joule of heat energy (during 12 hours). Therefore, a significant part of the available heat energy had to be transferred extremely rapidly from the lava flow to the substrate to thermally erode the channel. The corresponding heat current density (transferred energy/(time $\times$ channel surface)) amounts to $\sim 10^{5} \mathrm{~W} / \mathrm{m}^{2}$ ). Even with a temperature gradient on the order of $\sim 100 \mathrm{~K} / \mathrm{m}$ this corresponds to a heat conductivity of about $\sim 10^{3} \mathrm{~W} /(\mathrm{m} \cdot \mathrm{K})$.

This is rather unrealistic as can be seen by the following considerations. First, the huge heat transfer rate would have required a heat conductivity exceeding by far that of noble metals (e.g., for silver one has $\kappa_{\mathrm{Ag}} \approx 4 \times 10^{2} \mathrm{~W} /(\mathrm{m} \cdot \mathrm{K})$ [17] while basaltic lavas have $\kappa \sim 1$ $\mathrm{W} /(\mathrm{m} \cdot \mathrm{K})[\underline{6}, \mathbf{7}])$. Second, the noticeable heat loss would have increased viscosity leading to rapid deceleration and eventual standstill of the flow. This is in contradiction with the field observations. Finally, we have neglected heat loss, e.g., radiative heat loss and the formation of chill layers [7]. Thus, we conclude that thermal erosion cannot have played a dominant role in the channel formation.

Mechanical erosion hypothesis - Friction and wear are ubiquitous phenomena in everyday life. Their investigation in physics and engineering sciences, however, is a relatively young field [18, 19]. Mechanical erosion is one of the fundamental processes in geology, quantitatively studied, e.g., in the context of bedrock [20] and glacial [21] erosion. While such studies focus on the dynamics, based on field data for many examples, the aim of the present discussion is merely to argue that the formation of the Laghetto channel does comply with the fundamental concepts of abrasive wear.

The idea of purely mechanical erosion is imposed by the fact that strata in the channel banks are clearly distinguished (cf. Fig 2), indicating that the material was removed by "cutting" rather than by melting. The question is which mechanisms have acted and how they can be described quantitatively.

We propose the following intuitive picture. The erosive lava was a thick (up to $20 \mathrm{~m}$ ) autoclastic flow, i.e., a dense mixture of crystals and clasts, metric in size, suspended in a 
melt matrix. The mesh of clasts got dragged along the bed such that the irregular clast surfaces in contact with the bed could plough out grooves. These are the characteristics of abrasive and erosive wear processes [22].

It needs to be emphasized that abrasion and wear are rather complex phenomena and therefore hard to model at a 'microscopic' level (that is, by identifying a dominant mechanism and deducing, e.g., wear rates from dynamical equations and material properties, in particular if the process parameters cannot be defined as well as in systematic lab experiments). For this reason, phenomenological models that are well-established by empirical studies and describe whole classes of wear processes are of considerable importance. One of the simplest quantitative models to describe wear phenomena is Archard's law [18]. It establishes a phenomenological relation between the wear volume $W_{v}$, the normal load $F$, and the sliding distance $s$ :

$$
\frac{W_{v}}{s}=k \cdot \frac{F}{H}
$$

Here, $H$ denotes the hardness of the worn material proportional to the yield strength $\tau$ $(H \approx 3 \tau)$. Further, $k$ is the wear coefficient, i.e., a dimensionless constant which depends on the wear mechanism. Abrasive wear by sliding glaciers is described by similar models [21]. From the observations we can estimate $W_{v}, s, F$, and $H$. For a realistic model, $k$ should be 0.1 or smaller. The wear volume is $W_{v} \approx 10,000 \mathrm{~m}^{3}$. The sliding distance is the total length of the flow $(s \approx 2000 \mathrm{~m})$ and the load $F$ is the weight of the abrading lava along the length of the channel. With an average height of the flow of $10 \mathrm{~m}$ we find $F \approx 26,000 \mathrm{~m}^{3} \times 2800$ $\mathrm{kg} / \mathrm{m}^{3} \times 9.8 \mathrm{~m} / \mathrm{s}^{2} \approx 7.3 \times 10^{8} \mathrm{~N}$.

It is more difficult to estimate the hardness $H$ of the substrate since its yield strength is an exponential function of temperature. Note that these lava layers had been erupted only days or hours before. Their outer skin was black, but they were still incandescent inside. For cold lavas yield strength values are $\sim 10^{7} \mathrm{~Pa}$. According to Ref. [23] for a temperature of $1000{ }^{\circ} \mathrm{C}$ yield strength is larger than $10^{4} \mathrm{~Pa}$. For a likely temperature of $900{ }^{\circ} \mathrm{C}$ we interpolate values between $10^{5}$ to $10^{6} \mathrm{~Pa}$.

By inserting these numbers into Eq. (2) we obtain $k \approx 10^{-3} \ldots 10^{-2}$. This wear coefficient lies well within the typical range for abrasive and erosive wear processes $\left(k \sim 10^{-5} \ldots 10^{-1}\right)$ measured in tribology 22], confirming the intuitive picture for the erosive action of the lava flow.

Note that our estimate of $k$ and its relation to the wear mechanism is rather robust 
against variations due to uncertainties in the observations while thermal erosion remains excluded by several orders of magnitude.

In view of the strong support that the experimental observations provide for the idea of mechanical erosion, we may invert the argument: we may ask what theoretical predictions can be obtained from assuming this kind of erosive mechanism, and compare them with the results for thermal erosion. To this end, we rewrite Archard's law Eq. (2) in terms of characteristic quantities of the erosive process. We have already mentioned the sliding distance $s$, the height of the flow $h_{\mathrm{f}}$, the density of the lava $\rho$, the hardness $H$ of the substrate, and the constant $k$ which characterizes the wear mechanism. Further we introduce the width $w_{\text {fl }}$ of the flow (the latter is equal to the channel width $w_{\mathrm{ch}}$ ), the depth $d_{\mathrm{ch}}$ and the length $l_{\mathrm{ch}}$ of the channel. With $W_{v}=l_{\mathrm{ch}} w_{\mathrm{ch}} d_{\mathrm{ch}}$ and $F=\rho g l_{\mathrm{ch}} w_{\mathrm{fl}} h_{\mathrm{fl}} \cos \alpha$ (where $\alpha$ is the angle of the slope; for simplicity we will approximate $\cos \alpha \approx 1$ for moderate slopes) we obtain the relation

$$
d_{\mathrm{ch}}=\frac{k \rho g}{H} h_{\mathrm{fl}} s
$$

This equation can be further transformed by assuming that the flow rate per unit width $Q$ was constant for the duration $t_{\mathrm{fl}}$ of the flow. The result is

$$
V_{\text {mech }}=\frac{k \rho g}{H} Q
$$

where $V_{\text {mech }}$ denotes the velocity of mechanical erosion $V_{\text {mech }}=\frac{\mathrm{d}}{\mathrm{d} t_{\mathrm{fl}}} d_{\mathrm{ch}}$. This relation can be compared to Kerr's key result for the melting velocity $V_{\text {thermal }}$ due to laminar flows [7]

$$
V_{\text {thermal }} \propto\left(\frac{U}{h_{\mathrm{fl}, \text { thermal }}}\right)^{1 / 3},
$$

here $U$ denotes the surface velocity of the flow, and we have identified $d_{\mathrm{ch}} \equiv h_{\mathrm{fl}}$ for thermal erosion. While Eq. (5) implies, e.g., [7]

$$
V_{\text {thermal }} \propto Q^{1 / 9}, \quad h_{\mathrm{fl}, \text { thermal }} \propto Q^{1 / 3}
$$

our simple theory of mechanical erosion predicts

$$
V_{\text {mech }} \propto Q \propto h_{\mathrm{fl}, \text { mech }}
$$

Moreover, the substrate yield strength depends exponentially on the substrate temperature: $H=a \exp \left(-b T_{\text {subst }}\right)$ (with material constants $\left.a, b>0\right)$ and thus determines the temperature 
dependence of the ratio $V_{\text {mech }} / Q$ in Eq. (4) while for thermal erosion the ratio $V_{\text {thermal }} / Q^{1 / 3}$ depends on $T_{\text {subst }}$ via a rational function (the Stefan number, cf. Ref. 7]]).

Thus, our hypothesis leads to concrete predictions which are substantially different from those for thermal erosion, even without precise knowledge regarding the 'microscopic details' of the erosion mechanism. An important consequence of Eqs. (6) and (77) is that, with increasing flow rate $Q$, the velocity of mechanical erosion $V_{\text {mech }}$ grows much faster than that of thermal erosion. That is, for typical parameters of basaltic lava flows [7, 23] one may obtain velocities of mechanical erosion that are one or two orders of magnitude larger than those for thermal erosion due to laminar flows with the same flow rate. This fact may help to explain the observation of lava-channel formation even in the absence of very high eruption temperatures and long-lived, low-viscosity flows (cf. Ref. [13]).

Conclusions - The analysis presented here focused mainly on explaining the physical processes at the origin of the Laghetto channel. It may serve as a basic approach to mechanical erosion by lava flows. Further field studies and a better understanding of the erosive processes at the 'microscopic level' will help to refine the model. It is important to note that the observation of the channel formation described here represents a singular event. Nevertheless, similar features indicating mechanical erosion can be found in historical and prehistorical lava fields on Mount Etna and elsewhere [13]. Finally we mention that new aspects in channel formation processes on Earth may provide useful hints also for the assessment of erosion mechanisms in planetary environments such as lunar sinuous rilles and venusian canali [1, 3, [6].

Acknowledgments - The authors would like to thank R. Cristofolini and G. Ori for stimulating discussions. J.S. is supported by a Heisenberg fellowship of the German Research Foundation.

[1] G. Hulme, Mod. Geol. 4, 107 (1973).

[2] M.H. Carr, Icarus 22, 1 (1974).

[3] G. Hulme, Geophys. Surv. 5, 245 (1982).

[4] H.E. Huppert and R.S.J. Sparks, J. Petrol. 26, 694 (1985).

[5] D.A. Williams, R.C. Kerr, C.M. Lesher, J. Geophys. Res. 103, 27,533 (1998). 
[6] S.A. Fagents, R. Greeley, Bull. Volcanol. 62, 519 (2001).

[7] R.C. Kerr, J. Geophys. Res. 106, 26,453 (2001).

[8] H.E. Huppert, R.S.J. Sparks, J.S. Turner, N.T. Arndt, Nature 309, 19 (1984).

[9] J.B. Dawson, H. Pinkerton, G.E. Norton, D.M. Pyle, Geology 18, 260 (1990).

[10] H. Pinkerton, L. Wilson, G. Norton, Proc. Lunar Planet. Sci. Conf. XXI, 964 (2000).

[11] J. Kauahikaua et al., J. Geophys. Res. 103, 27,303 (1998).

[12] R. Greeely, S.A. Fagents, R.S. Harris, S.D. Kadel, D.A. Williams, J. Geophys. Res. 103, 27,325 (1998).

[13] D.A. Williams, S.D. Kadel, R. Greeley, C.M. Lesher, and M.A. Clynne, Bull. Volcanol. 66, 168 (2004).

[14] F.J. Ciesla, L. Keszthelyi, Proc. Lunar Planet. Sci. Conf. XXXI, 1647 (2000).

[15] S. Calvari and H. Pinkerton, J. Volcanol. Geotherm. Res. 132, 225 (2004).

[16] M.S. Ghiorso and R.O. Contrib. Mineral. Petrol. 119, 197 (1995).

[17] N.W. Ashcroft and N.D. Mermin, Solid State Physics, (Saunders, Philadelphia, 1976).

[18] F.P. Bowden, D. Tabor, The Friction and Lubrication of Solids, (Clarendon, Oxford, 1964).

[19] T.F.J. Quinn, Physical Analysis for Tribology , (Cambridge University Press, Cambridge, 1991).

[20] W.E. Dietrich et al., Geomorphic transport laws for predicting landscape form and dynamics. Prediction in Geomorphology. In: Geophysical Monograph 135, P. Wilcock, R. Iverson (Eds.), in press.

[21] D. Drewry, Glacial Geologic Processes, (Edward Arnold, London, 1986).

[22] K.-H. Zum Gahr, Tribology Int. 31, 587 (1998).

[23] J.H. Fink (Ed): Lava Flows and Domes, (Springer, Berlin, 1990). 
Fig. 1: Aerial view of the Laghetto eruptive vent. The channel can be seen to the left of the pyroclastic cone. As indicated the erosional feature (head of the channel) starts immediately at the emission vent. Its length $(220 \mathrm{~m})$ gives the scale of the picture. In the upper part, there is the lava field produced by the eruption of 2001.

Fig. 2: The right bank of the channel. In the foreground the bottom of the channel can be seen. It is covered by the eroding lava flow. The incision of the channel renders visible the layered structure of the substratum. It is important to note that these layers erupted few days or hours before were still rather soft and plastic. This is displayed by the uppermost layer folded inside the channel just after its incision.

Fig. 3: Cartoon showing the mechanical erosion of the channel (not in scale). a) Near the vent (less than $500 \mathrm{~m}$ ), the preflow terrain consisted of thin layers of hot lava. b) While moving down the slope the autoclastic lava flow carved out the soft parts of the substrate. c) Situation after the flow had stopped. The channel floor is partially covered by lava of the eroding flow. 


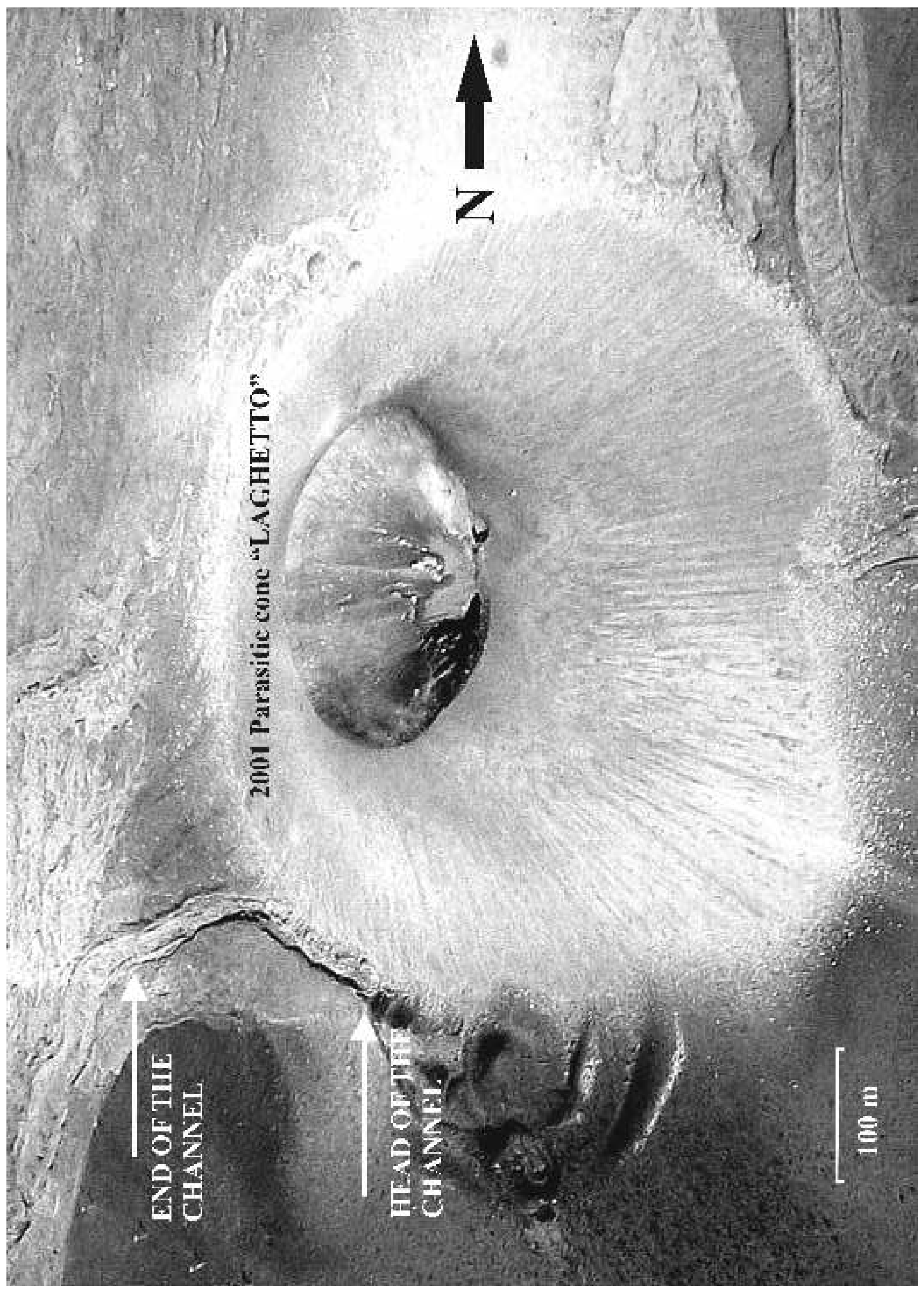




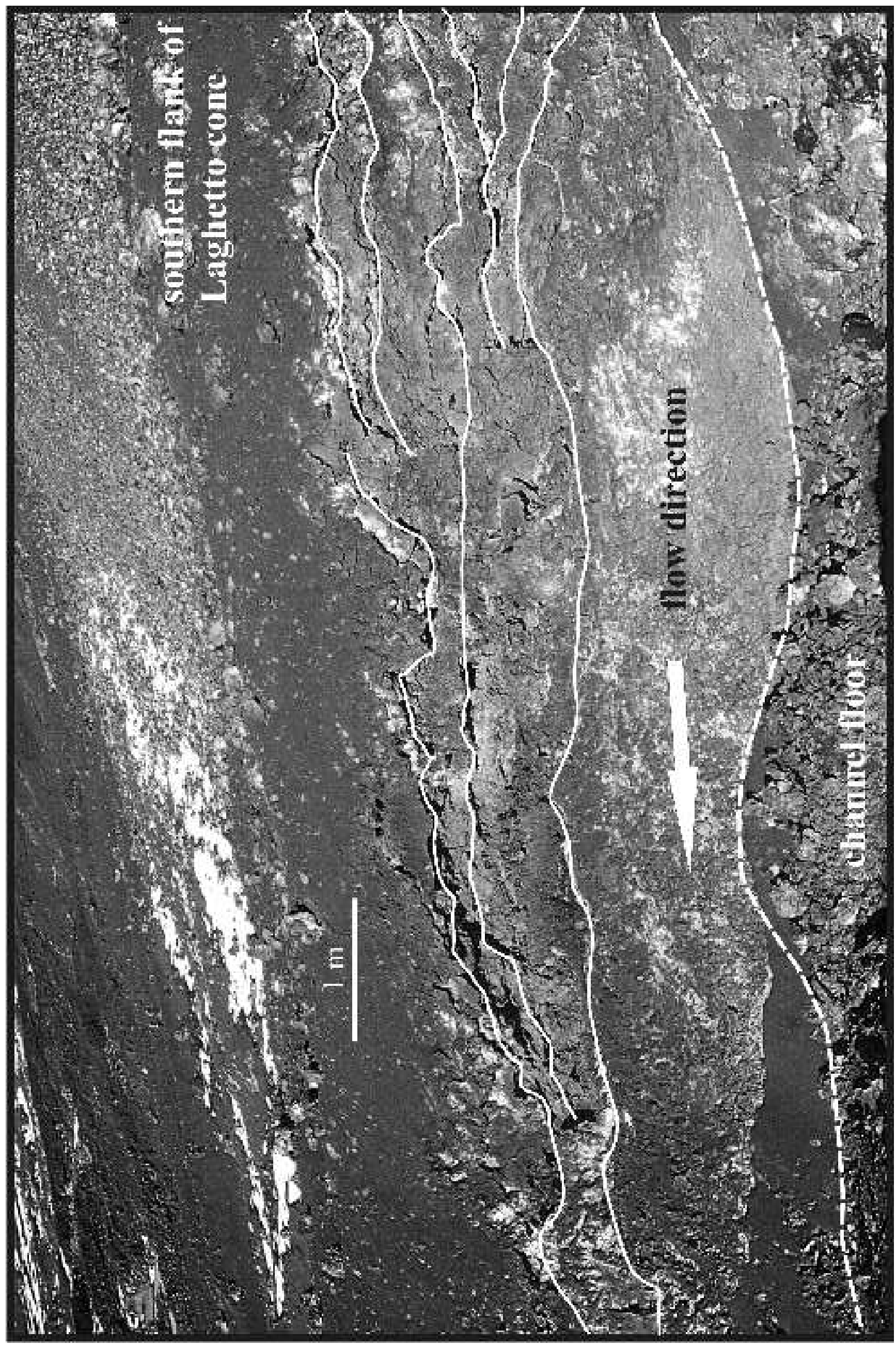




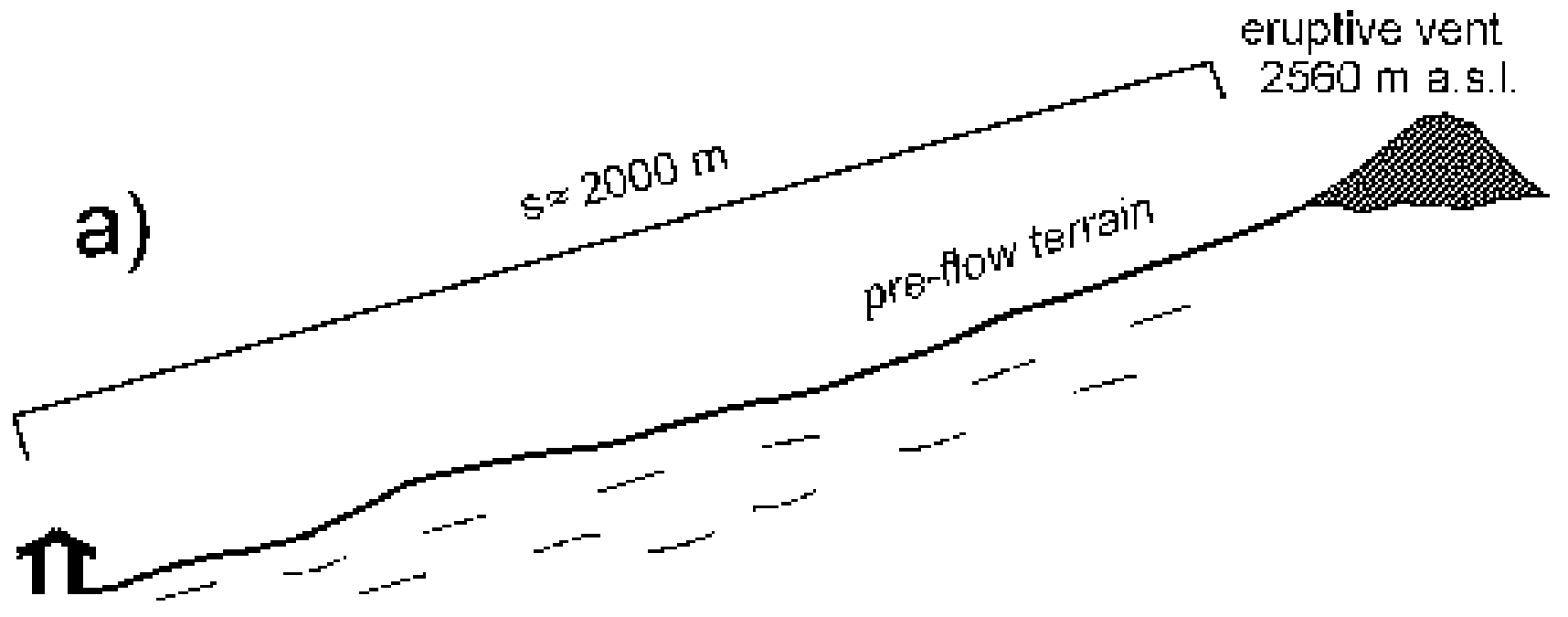

Rifugio Sapienza
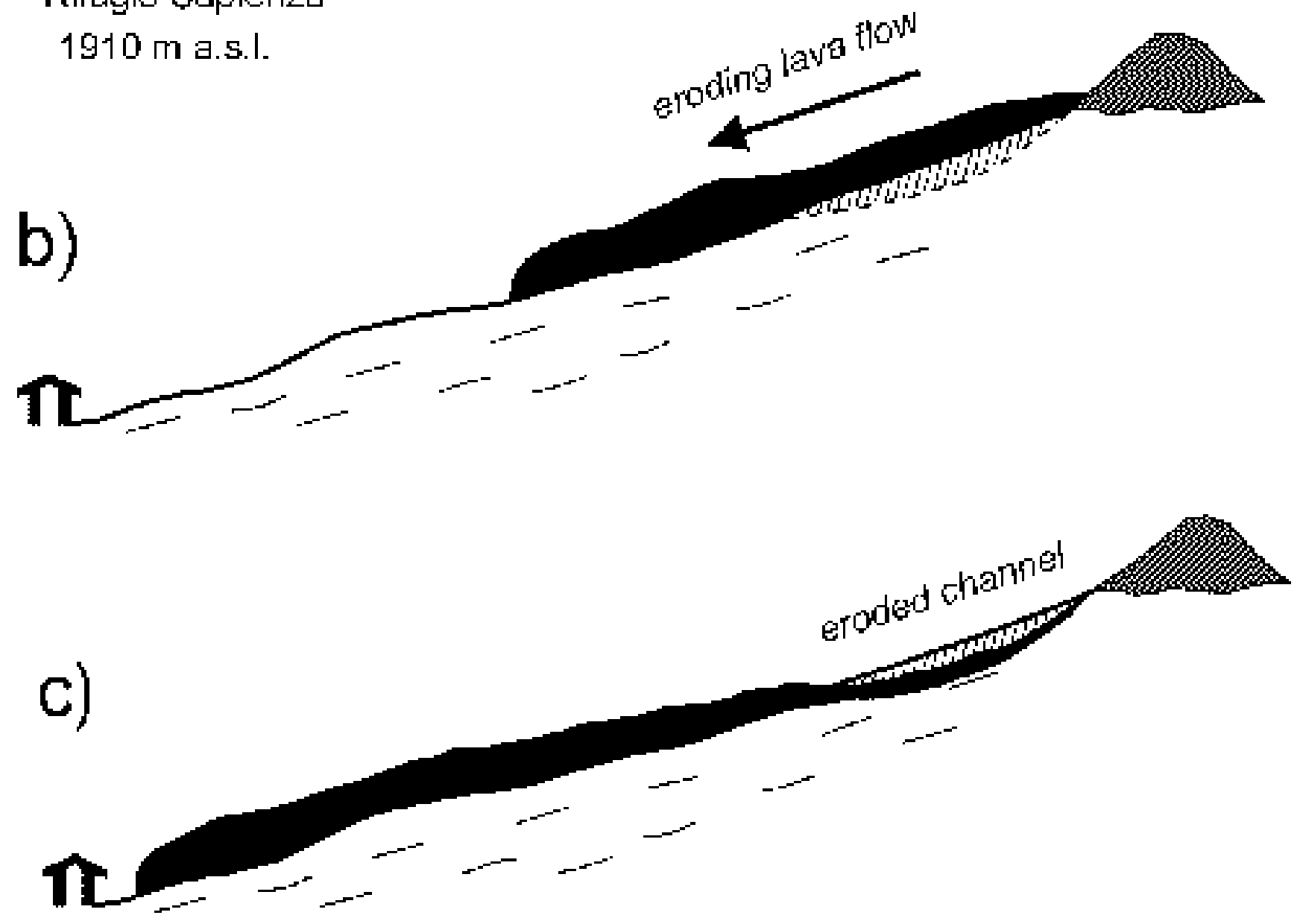